\title{
Dissolution Highlights from the 2011 AAPS Annual Meeting in Washington, D.C.
}

\author{
Vivian Gray ${ }^{1}$, Nikoletta Fotaki ${ }^{2}$, and Gregory P. Martin ${ }^{3}$ \\ 'V. A. Gray Consulting, Hockessin, DE, USA \\ ${ }^{2}$ University of Bath, UK \\ ${ }^{3}$ Complectors Consulting, Pottstown, PA, USA
}

e-mail:vagray@rcn.com

T he 2011 AAPS Annual Meeting provided several programs on dissolution and related topics, including a Roundtable on IVIVIC, a sunrise session on bioequivalence studies, and meetings of the In Vitro Release and Dissolution Testing Focus Group (IVRDT FG). There was also a workshop on the topic of Oral Dosage Forms, which will be reported on in the May issue of Dissolution Technologies. In addition to programming directed specifically toward dissolution, there were many other programs of interests to pharmaceutical scientists. Some highlights were:

- Challenges and Opportunities for Designing Quality in Generic Drug Development: Are We There Yet?

- Impact of the Implementation of Well Designed Electronic Laboratory Notebooks on Laboratory Functions

- Linking Quality to Safety and Efficacy

- Impurities

- A New Era for Post-Approval Changes: Recent Developments from FDA and EMA

"In Vitro in Vivo Correlations-Real-World Case Studies of Successes and Failures" was discussed in a roundtable session. Alger Salt from GSK talked about the assessment of the feasibility of establishing an IVIVC. He posed the question on how we could respond to an IVIVC development request, and a case study was discussed. He presented a scenario in which a request for the development of IVIVC to justify the proposed dissolution specifications for an oncology drug was questioned. The associated risks with conducting an IVIVC study (dosing restricted to patient, long recruitment period, risk to patients by altered release rates of the formulation, design of the study, probability of success for IVIVC) were noted, and the approach followed for evaluating the chance for a successful IVIVC was described. The study used absorption rate analysis, which revealed that drug absorption is largely controlled by the gastric emptying rate and that dissolution occurs only in the stomach. The hypothesis was bolstered with data showing that in vivo dissolution was similar and independent of the formulation. It was concluded that the chance for demonstrating IVIVC would be very low and that IVIVC would have a high rate of success when dissolution rate is a function of formulation parameters.

Filippos Kesisoglou from Merck discussed the challenges in developing IVIVC for an immediate-release
(IR) product. He began by addressing some common questions on the development of IVIVCs for IR products (i.e., if an IVIVC should be expected, the level of IVIVC that would be possible, the methodology that can be used, and the regulatory acceptance of different methods). A case study for an IR formulation of a BCS Class II compound was presented. Upon successful development of a multiple level C correlation, a level A correlation was attempted. A traditional level A IVIVC through standard deconvolution methodology and a level A IVIVC based on absorption modeling were developed. The traditional approach was not fully "compliant" with prediction errors as set in ER IVIVC guidance, and the necessity of parameter fitting for the second approach was noted. The importance of the time-scaling factor in both cases was highlighted. In his conclusion, he stated that Level C (or multiple level C) can be obtained for IR formulations and provides good differentiation of release rates, even though changes may be restricted only to $C_{\max }$ and not to AUC (especially for BCS Class II drugs). He noted that the development of level A IVIVC is more challenging. He posed the question about the criteria for declaration of an IVIVC and if the ER IVIVC guidance criteria should be followed.

The use of IVIVC of Extended-Release Products for the prediction of generic drug performance was discussed by Tahseen Mirza from FDA. He presented a case study to determine if an IVIVC model can predict in vivo profiles of (1) formulations that vary in excipients and manufacturing process but belong to the same mechanism of release and (2) formulations that belong to a different mechanism of drug release (to challenge the robustness of the IVIVC model). An innovator and five generic formulations of a BCS Class I compound from the FDA were chosen. An IVIVC model was developed through the traditional approach (deconvoluted in vivo absorption vs in vitro dissolution) and through simulation (with Gastro Plus). Internal (formulation data used in the model development) and external validation (formulation data used to challenge the model) revealed that the IVIVC model might be applicable to different formulations if the principle of drug release is similar.

Then the highlights of the results from a survey on "IVIVC: Critical Variables for Success" organized by the AAPS In Vitro Release and Dissolution Testing Focus Group and the QbD \& Product Performance Focus Group were presented by Nikoletta Fotaki from University of Bath. 
The objectives of the survey were to describe the current status and to identify the critical variables for the development of IVIVC. This included the use and success rates of IVIVCs, the main difficulties in pursuing an IVIVC, the use of IVIVC for dissolution specification setting, and the tools and methods used for the development of IVIVCs. IVIVCs for BCS Class II and BCS Class IV immediate-release compounds were addressed.

Discussion followed the presentations. Topics included appropriate dissolution methods, the acceptability of noncompendial dissolution apparatus, the use of Design of Experiments (DOE), and the feasibility of IVIVC for BCS Class II drugs in immediate-release formulations.

\section{A Sunrise Session: Achieving Bioavailability and Streamlining Bioequivalence Studies for Oral Drug Products.}

This session was moderated by Jennifer Sheng, Novartis, and John Crison, BMS. There were two presentations. The first was given by Gordon Amidon, University of Michigan, on the topic "New Era of Biopharmaceutics Understanding of BA and BE." He reviewed where we have come with $\mathrm{BCS}$ and the addition of the BDDCS to our understanding. He reiterated that biorelevant dissolution methods are the best hope for testing for bioequivalence rather than expensive biostudies. The second speaker was Lawrence $\mathbf{Y u}$ of the FDA, and his topic was "Current Status and Future Perspective of Regulation of BA and BE: Linkage to the Scientific Insights." Dr. Yu first discussed the difficulty of determining $B E$ with highly variable drugs. There have been many publications from FDA on the subject of BE standards for highly variable drugs and narrow therapeutic drugs. The advisory panel on the subject has recommended that the $\mathrm{BE}$ standards be stricter and that future research into pharmacodynamics (PD) and the reasons for therapeutic failures be undertaken. He also discussed the issues with dissolution testing and emphasized that the dissolution test alone is not sufficient to assure product quality and bioavailability and that the IVIVC is necessary.

\section{IVRDT FOCUS GROUP BUSINESS MEETING}

The annual meeting of the IVRDT Focus Group was held on Wednesday, October 26. The meeting was attended by about 30 members. Chair Alger Salt presented the 2011 Focus Group Accomplishments, which include two international Outreach Workshops on Dissolution (these workshops are featured in this issue of Dissolution Technologies), two newsletters, collaboration with the QbD \& Product Performance Focus Group on an excellent program on IVIVC held at the AAPS Annual Meeting, and initial efforts to put together an e-Learning course on Dissolution. Plans for 2012 include completion of the e-Learning course, collaboration with the Nutraceuticals and Natural Product Chemistry Focus Group on programming for the 2012 AAPS Annual Meeting, and an international Outreach Workshop on Bioequivalence and Dissolution in South Africa. We were pleased to announce that Vivian Gray, Founder of the IVRDT Focus Group, received the APQ Service Award for 2011. The meeting ended with a call for topics for 2012 and an invitation for new members to join. More information about the Focus Group, including some recent presentations and the newsletter, can be found on the AAPS website.

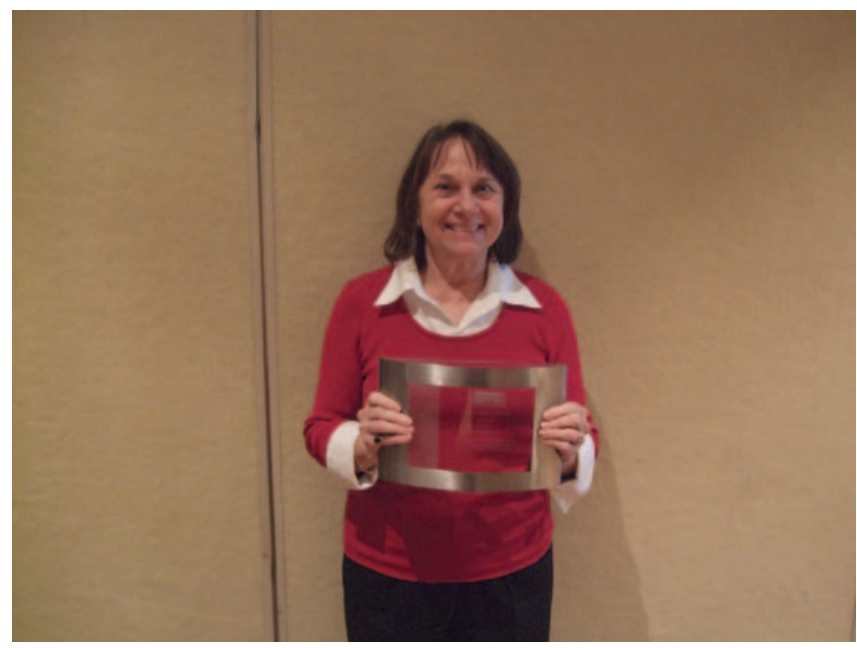

Vivian Gray - APQ Service Award 